\title{
RESEARCH
}

\section{EVALUATION OF DIGOXIN LEVELS IN ELDERLY PATIENTS IN A TRAINING HOSPITAL: AN 11-YEAR FOLLOW-UP}

Turkish Journal of Geriatrics

DOI: 10.31086/tjgeri.2020.144

2020; 23(1): 108-117

- İsmail YILMAZ1 D

- Sibel BiLGíli2 iD

. Giray BOZKAYA ${ }^{2}$
CORRESPONDANCE

\section{İsmail YILMAZ}

Health Sciences University, Izmir Bozyaka Training and Research Hospital, Clinical Pharmacology and Toxicology, Biochemistry Department, Izmir, TURKEY.

Phone: +90532285958

e-mail: driyilmaz@yahoo.com

\section{A}

Introduction: Digoxin monitoring is important in the elderly because of the toxicity risk. The aim of this study was to evaluate the orders made by different units for digoxin monitoring in the elderly.

Materials and Method: Digoxin orders and results in patients aged over 65, patients' demographic characteristics, such as age and gender, the unit from which the order was made, and patients' pre-diagnoses were retrospectively recorded. Patients were divided into young-old, middle-old, and very-old groups, and the results were divided into subtherapeutic, therapeutic, and toxic levels.

Results: The percentage of young-old, middle-old, and very-old patients was $39.4 \%, 45.3 \%$, and $15.3 \%$, respectively. Women were more $(64.3 \%)$ and their median age and digoxin concentrations were higher $(p<0.001)$. Outpatients' median digoxin concentrations were lower than inpatients' median digoxin concentrations $(p<0.001)$. A proportion of $46.6 \%$ of all digoxin levels were therapeutic; $29.2 \%$ were subtherapeutic; and $24.2 \%$ were toxic. Subtherapeutic levels in males and toxic levels in females predominated $(p<0.001)$. Toxic levels were higher in inpatients, and subtherapeutic levels were higher in outpatients $(p<0.001)$. The highest rate of therapeutic levels was observed in young-old patients (48.7\%), while toxic levels were observed most frequently in very-old patients $(28.1 \%)$. The highest number of digoxin orders in elderly patients was from the emergency department (44.2\%).

Conclusion: In elderly patients, more than half of the serum digoxin orders were inappropriate. These results significantly changed according to gender, outpatient or inpatient status, age, and the ordering unit. Changes in digoxin pharmacokinetics, especially in the geriatric period, require physicians to be vigilant of subtherapeutic drug concentrations.

Keywords: Aged; Geriatrics; Digoxin; Drug monitoring.

Received: 29/01/2020

Accepted: 28/02/2020

${ }^{1}$ Health Sciences University, Izmir Bozyaka

Training and Research Hospital, Clinical

Pharmacology and Toxicology, Biochemistry

Department, Izmir, TURKEY.

2 Health Sciences University, Izmir Bozyaka

Training and Research Hospital, Biochemistry

Department, Izmir, TURKEY. 


\section{INTRODUCTION}

The prevalence of heart failure and the risk of atrial fibrillation increase with age (1). Digoxin can be an appropriate agent to treat systolic dysfunction, high ventricular rate, and atrial fibrillation in the elderly. The elderly population appears to experience comparable symptom improvement and hospitalization reduction to younger populations from the use of digoxin for heart failure management $(1,2)$. However, digoxin has a complex pharmacokinetic profile, narrow therapeutic range, and high risk of toxicity. Also, pharmacological properties of digoxin may vary significantly between men and women (3). According to the Digitalis Investigation Group, the risk of all-cause mortality and all-cause hospitalization was lower than placebo in patients with digoxin concentrations of $0.5-0.9 \mathrm{ng} / \mathrm{mL}$, and mortality increased significantly to $12 \%$ in those with digoxin concentrations $\geq 1.2 \mathrm{ng} / \mathrm{mL}$. Therefore, serum levels need to be monitored to optimize therapeutic performance, detect noncompliance, and reduce toxicity $(4,5)$. Digoxin toxicity may mimic certain symptoms of heart disease, and measuring the plasma concentration in cases in which toxicity is suspected may help confirm a diagnosis. The recognition of digoxin toxicity is more difficult in elderly patients than in younger patients (6). When digoxin is used in elderly patients, the specific geriatric pharmacokinetics of the drug must be considered. Changes in serum digoxin levels require further evaluation in elderly patients because of significant risks specific to comorbid diseases, sex, electrolyte disturbances, and drug interactions. In addition, elderly patients are especially susceptible to digoxin toxicity $(2,5)$. Patients over 70 years of age may show clinical signs of digoxin toxicity despite having digoxin concentrations within the recommended therapeutic range. Increased age is most likely associated with enhanced susceptibility to digoxin toxicity due to unknown pharmacodynamic changes $(7,8)$. Digoxin is in the top five causes of emergency department admissions due to adverse drug reactions (9), and it is among the top three drugs that cause emergency department admissions in patients over 65 years (10).

Serum digoxin concentration is the most commonly ordered therapeutic drug monitoring test globally (11). Despite many clinical trials, studies evaluating digoxin orders specifically in the elderly are uncommon. The aim of this study was to evaluate the orders made by different units in elderly patients aged over 65 years and to evaluate the eleven-year data of these results according to age groups, sex, and inpatient or outpatient status.

\section{MATERIALS AND METHOD}

\section{Study design}

This was a retrospective, descriptive study. This study began after its approval by the Local Ethics Committee for Non-Interventional Research and was performed in accordance with the Declaration of Helsinki. Between 1 January 2008 and 31 December 2018, the data for serum digoxin orders in patients over the age of 65 sent to the Medical Biochemistry Laboratory in Health Sciences University Izmir Bozyaka Training and Research Hospital to be studied from different departments and polyclinics were collected from the hospital electronic medical record and analysed. The demographic characteristics, such as age and sex, of the patients, unit from which the order was made, pre-diagnosis codes of the patients according to the International Classification of Diseases-10 (ICD-10) codes, order time, and serum digoxin levels were recorded. In the study, since there were multiple digoxin measurements on behalf of the same patient, a demand-based evaluation was performed in both inpatients and outpatients. Patients older than 65 years were divided into young-old (65-74 years), middle-old (75-84 years), and very-old (85 years and older) groups, and the results were grouped as subtherapeutic $(<0.8 \mathrm{ng} / \mathrm{mL})$, therapeutic $(0.8-2$ $\mathrm{ng} / \mathrm{mL})$, and toxic (>2 $\mathrm{ng} / \mathrm{mL}$ ) levels. 


\section{Methods}

Serum samples were analysed spectrophotometrically (Beckman-Coulter, Digoxin, OSR6404) with the Olympus AU-680 autoanalyzer (Beckman-Coulter, Fullerton, USA) in the Medical Biochemistry Laboratory, and the results are expressed in $\mathrm{ng} / \mathrm{dL}$. Two levels of internal quality control were used daily at each shift change. In addition, external quality control was performed monthly. The detection limit of the method used in the study was $0.14 \mathrm{ng} / \mathrm{dL}$, and the maximum measurement value was $5 \mathrm{ng} / \mathrm{dL}$. The samples with higher concentrations were diluted according to the manufacturer's instructions and re-measured.

\section{Statistical analysis}

Data are presented as frequencies ( $n$ ) and percentages (\%) or medians and interquartile ranges. The medians were compared using the MannWhitney U-test, as applicable, after checking normality using the Kolmogorov-Smirnov test. Test results with $p$ values $<0.05$ were determined to be statistically significant.

\section{RESULTS}

In the study, 10810 digoxin orders of 4420 patients from all age groups were evaluated. While the median age of the patients was 72.6 (12.4-110 years), 2.44 orders were made per patient. The main content of our study was 8390 digoxin orders of 3502 patients aged 65 and over, and it was observed that 2.39 digoxin orders were made per patient in the elderly group. According to order-based evaluation the median age of all elderly patients was 77.3 years (72-82.2 years). Among all patients, 3307 were young-old (39.4\%); 3797 were middle-old (45.3\%); and 1286 were very-old (15.3\%). The median age of the young-old patients was 70.7 years (68.2-73 years); that of the middle-old was 79.6 years (77.4-81.9 years); and that of the very-old was 87.8 years (86-90.8 years). The results included 5392 (64.3\%) levels in women and 2998 (35.7\%) levels in men. In men, the median digoxin level was $1.18 \mathrm{ng} / \mathrm{mL}(0.61-1.81 \mathrm{ng} / \mathrm{mL})$, and the median age was 76.2 years (70.7-81.5 years). In women, the median digoxin level was $1.3 \mathrm{ng} / \mathrm{mL}(0.72-2.06 \mathrm{ng} /$ $\mathrm{mL})$, and the median age was 77.8 years (72.6-82.8 years). Both of these variables were statistically significant $(p<0.001)$. Outpatient orders $(4605,54.9 \%)$ were higher than inpatient orders $(3785,45.1 \%)$. In outpatients, the median digoxin level was 1.08 $\mathrm{ng} / \mathrm{mL}(0.53-1.74 \mathrm{ng} / \mathrm{mL})$, and the median age was 77.3 years (71.9-82.6 years). In inpatients, the median digoxin level was $1.5 \mathrm{ng} / \mathrm{mL}(0.89-2.19 \mathrm{ng} /$ $\mathrm{mL})$, and the median age was 77.3 years (72.2-81.9 years). The difference in the median digoxin levels between outpatients and inpatients was statistically significant $(p<0.001)$, but no significant difference was found for age or gender $(p>0.05)$. While $6282(74.9 \%)$ of all pre-diagnoses entered into the hospital information system were cardiac-related or digoxin-related, 2108 (25.1\%) were non-cardiac, irrelevant diagnoses.

The median digoxin level for all patients was $1.26 \mathrm{ng} / \mathrm{mL}$ (0.68-1.98 ng/mL). In young-old, middle-old, and very-old patients, the median digoxin levels were $1.15 \mathrm{ng} / \mathrm{mL}(0.64-1.81 \mathrm{ng} / \mathrm{mL}), 1.33 \mathrm{ng} /$ $\mathrm{mL}(0.71-2.06 \mathrm{ng} / \mathrm{mL})$, and $1.4 \mathrm{ng} / \mathrm{mL}(0.7-2.17 \mathrm{ng} /$ $\mathrm{mL})$, respectively. According to age groups, there was a significant difference in the mean serum digoxin levels between the young-old groups and the other groups $(p<0.001)$, but the difference between middle-old and very-old groups was not significant ( $p>0.05$ ). Among the digoxin levels, $46.6 \%$ (3908) were therapeutic; $29.2 \%$ were subtherapeutic (2453); and 24.2\% (2029) were toxic. Toxic levels were more frequent in women than in men (26.5\% and $20 \%$, respectively), and subtherapeutic levels were more frequent in men than in women (32.4\% and $27.5 \%$, respectively). The relationship between subtherapeutic and toxic levels and gender was statistically significant $(p<0.001)$. Therapeutic levels were similar in men and women $(46 \%$ and $47.6 \%$, respectively). The distribution of therapeutic levels by gender is shown in Figure 1. The subtherapeutic level rate was higher in outpatients 
Figure 1. The therapeutic level rates of digoxin by gender in elderly. Data are presented percentages $(\%) .{ }^{*} \mathrm{p}<0.001$ between female and male.

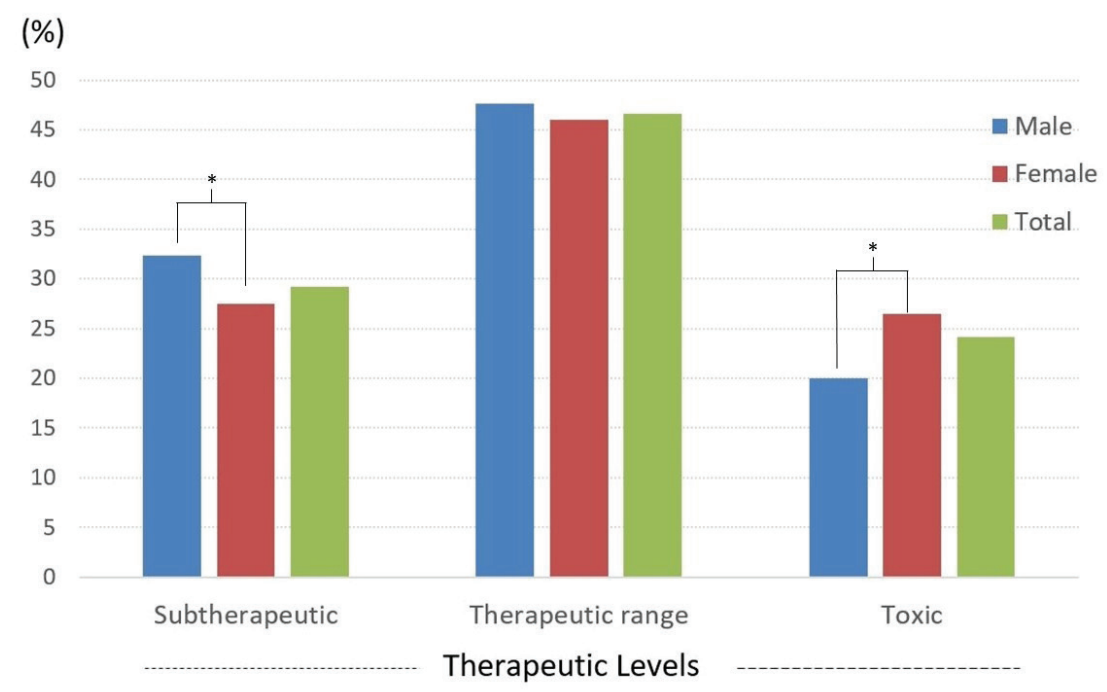

Figure 2. The therapeutic level rates of digoxin by outpatients and inpatients in elderly. Data are presented percentages (\%). ${ }^{*} p<0.001$ between outpatients and inpatients.

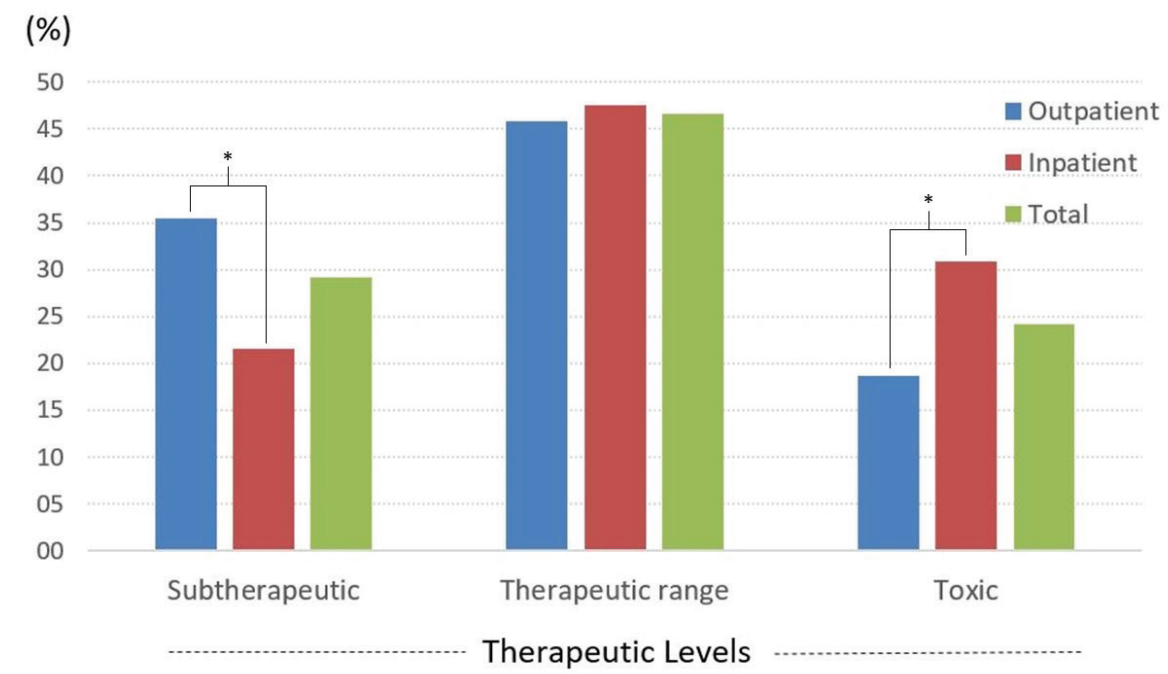

than in inpatients $35.5 \%$ and $21.6 \%$, respectively). In contrast, inpatients had higher therapeutic (47.6\% vs. $45.3 \%)$ and toxic (30.8\% vs. $18.7 \%$ ) levels than outpatients. The relationship between inpa- tients and outpatients and therapeutic levels was significant $(p<0.001)$. The distribution of all outpatient and inpatient orders according to therapeutic levels is shown in Figure 2. 
When the age groups were examined according to therapeutic levels, results within the therapeutic range were more frequently observed in the young-old patients (48.7\%), followed by the middle-old (45.6\%) and very-old (44\%) patients. Toxic levels were more frequently observed in the very-old patients $(28.1 \%)$, followed by the middle-old (26.7\%) and young-old (19.8\%) patients. The rate of subtherapeutic levels was similar between the middle-old and very-old groups (27.7\% and $27.9 \%$, respectively), and the rate was $31.5 \%$ in young-old patients. There was a significant difference in the rate of therapeutic levels between the young-old group and the other groups ( $p<0.001$ ), but there was no significant difference in this vari- able between the middle-old and very-old groups ( $p>0.05$ ). Table 1 shows the distribution for the frequency of the young-old, middle-old, and very-old patients who had therapeutic levels.

When the elderly patients from units that ordered digoxin were examined, the first three were the emergency department with 3711 orders (44.2\%), coronary intensive care unit with 1585 orders $(18.9 \%)$, and the internal medicine inpatient unit with 1561 orders (18.6\%). These units were followed by the internal medicine outpatient clinic, cardiology outpatient clinic, and other intensive care units. The patient distribution and rates of orders by unit are shown in Figure 3. Middle-old patients were the majority of patients in the three

Table 1. Distribution of young-old, middle-old and very-old patient orders according to therapeutic level.

\begin{tabular}{|l|r|r|r|r|r|r|r|r|}
\hline \multirow{2}{*}{ Age(Year) } & \multicolumn{2}{|c|}{ Subtherapeutic } & \multicolumn{2}{|c|}{ Therapeutic range } & \multicolumn{2}{|r|}{ Toxic } & \multicolumn{2}{|c|}{ Total } \\
\cline { 2 - 8 } & $\mathbf{n}$ & $\%$ & $\mathbf{n}$ & $\%$ & $\mathbf{n}$ & $\%$ & $\mathbf{n}$ & $\%$ \\
\hline Young-old & 1042 & 31.5 & 1609 & $48.7 *$ & 656 & 19.8 & 3307 & 100 \\
\hline Middle-old & 1052 & 27.7 & 1733 & 45.6 & 1012 & 26.7 & 3797 & 100 \\
\hline Very-old & 359 & 27.9 & 566 & 44 & 361 & 28.1 & 1286 & 100 \\
\hline
\end{tabular}

Data are presented as frequencies $(n)$ and percentages (\%). ${ }^{\star} p<0.001$ between young-old group and the other groups in the rate of therapeutic levels.

Figure 3. Digoxin orders according to clinics.

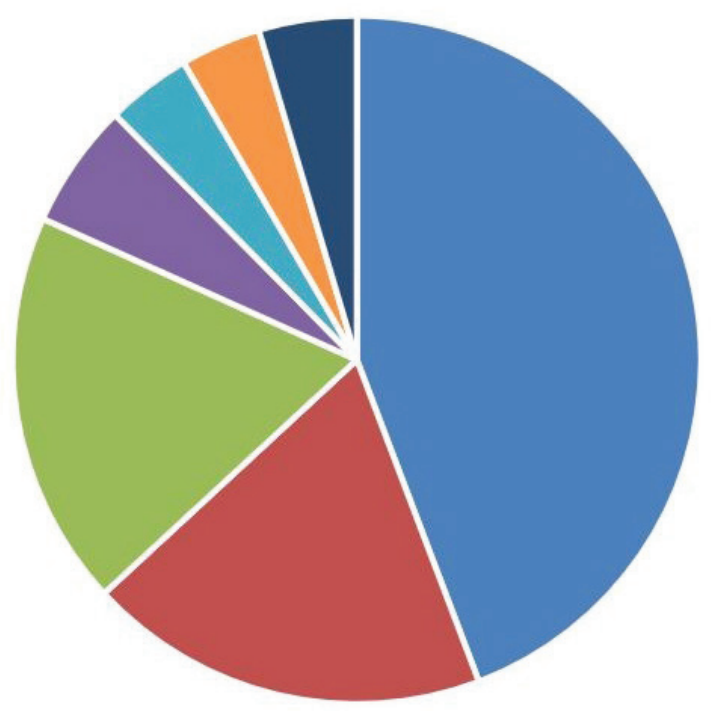

- Emergency

- Coronary

- Internal medicine

- Internal medicine polyclinics

- Cardiology polyclinics

- Other intensive care

- Other clinics 
Table 2. Age distribution of emergency, coronary, internal medicine and other clinics.

\begin{tabular}{|l|r|r|r|r|r|r|r|r|}
\hline \multirow{2}{*}{ Age(Year) } & \multicolumn{2}{|c|}{ Emergency } & \multicolumn{2}{r|}{ Coronary } & \multicolumn{2}{|r|}{ Internal medicine } & \multicolumn{2}{|r|}{ Other clinics } \\
\cline { 2 - 9 } & $\mathbf{n}$ & $\%$ & $\mathbf{n}$ & \% & $\mathbf{n}$ & \% & $\mathbf{n}$ & \% \\
\hline Young-old & 1327 & 35.8 & 590 & 37.2 & 616 & 39.5 & 774 & 50.5 \\
\hline Middle-old & 1660 & 44.7 & 745 & 47 & 768 & 49.2 & 624 & 40.7 \\
\hline Very-old & 724 & 19.5 & 250 & 15.8 & 177 & 11.3 & 135 & 8.8 \\
\hline Total & 3711 & 100 & 1585 & 100 & 1561 & 100 & 1533 & 100 \\
\hline
\end{tabular}

Data are presented as frequencies $(\mathrm{n})$ and percentages (\%).

Figure 4. The distribution of emergency, coronary intensive care and internal medicine service orders according to therapeutic level rates in elderly. Data are presented percentages (\%).

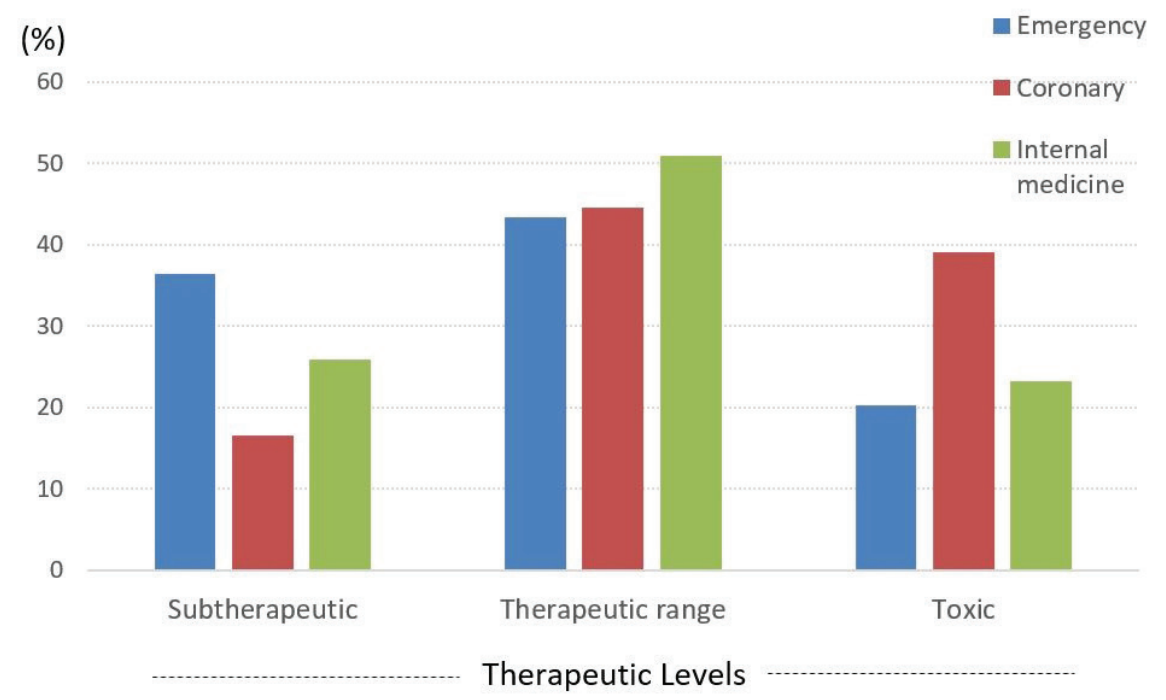

units with the most orders $(44.7 \%, 1660 ; 47 \%, 74$; and $49.2 \%, 768$, respectively). These patients were followed by young-old and very-old patients. The age distribution of all three units' orders is shown in Table 2. While the digoxin levels for emergency department orders were mostly in the therapeutic range $(43.3 \%, 1608)$, subtherapeutic levels were also noted $(36.4 \%, 1352)$. Of the coronary intensive care unit levels, $44.5 \%$ (706) were therapeutic, and $39 \%$ (618) were toxic. In the internal medicine unit, $50.9 \%$ (794) of all levels were therapeutic, while the rate of subtherapeutic and toxic levels were similar. The distribution of orders from the emergency department, coronary intensive care unit, and internal medicine unit according to therapeutic levels is shown in Figure 4. 


\section{DISCUSSION}

Studies show that a significant proportion of patients with serum digoxin level orders is composed of patients aged 65 years or older $(1,12)$. Among the digoxin orders in all age groups, the orders in patients over 65 years were $77.6 \%$ in our study. Orders were especially made in the middle-old patients, followed by young-old patients at a similar rate. Similarly, Ozturk and Cuhadar stated that the number of orders in middle-aged patients was higher than in other elderly people, and mean digoxin levels were higher in the middle-aged group (12). In our study, however, the median digoxin levels were significantly higher in the very-old group than in the middle-old group.

The correlation between gender and heart failure is much stronger in women than in men. This correlation leads to increased heart failure and, therefore, more digoxin use in women (13). The fact that valve diseases and rhythm disorders are more common in women (14) and that mortality occurs at a later age compared to men may cause digoxin use to appear relatively higher in women. The 1.8-fold higher rate in older women observed in our study compared to older men supports this theory and is in accordance with the results of many studies in the literature $(12,14-18)$.

In our study, less than half of all digoxin levels were within therapeutic limits; one-third of elderly patients had subtherapeutic levels; and approximately one-fourth were exposed to toxic levels. A few studies involving only the elderly and most studies evaluating all age groups suggest that the number of patients with therapeutic levels was low in our study and there is both insufficient patient adherence and follow up (11, 15, 16, 18-20). In a study by Grzesk et al., in which 3037 digoxin orders in patients over 60 years of age were evaluated, the therapeutic level rates were similar to the subtherapeutic level rates, and the toxic level rates were $13 \%$ lower than ours (19). In the same study, the mean serum digoxin levels in women were significantly higher than in men. Limon et al. stated that there was no significant association between digoxin poisoning and gender in a study population composed of patients, mostly women, with digoxin poisoning (17), while Canas et al. claimed that the percentage of patients with high levels of digoxin was significantly greater among men than among women (4). In our study, the mean digoxin levels in older women were significantly higher than those in older men. Dalaklioglu also stated that toxic levels are higher in women than in men, which contrasts the results of Canas et al. (16). Women have an approximately $10 \%$ lower glomerular filtration rate than men when normalised for body surface area. This difference might explain the observed 12 to $14 \%$ lower digoxin clearance in women than in men, since digoxin is primarily renally eliminated. Differences in blood serum digoxin levels between men and women may be due to these pharmacokinetic properties. This difference may lead to an increased risk of toxicity in women and an increase in subtherapeutic levels in men, as was observed in our study.

Although a distinction in the number of orders in outpatients and inpatients has been made in few published studies, digoxin orders are predominantly made in inpatients in the elderly $(15,19)$. In our study, the higher rate of outpatient orders may be due to the fact that the emergency department orders comprised $44.2 \%$ of all the orders. The mean digoxin levels were significantly higher in inpatients than in outpatients. Also, the rate of toxic levels was particularly high in inpatients. Grzesk et al. described this result in their study with therapeutic levels similar to ours by using intravenous treatment in addition to oral treatment in inpatients (19). In our study, the rate of subtherapeutic levels was higher in outpatients. Bernard et al. reported that $41 \%$ of digoxin results were subtherapeutic in their outpatients with an average age of 64 years and attributed this finding to collecting samples prior to the complete distribution of digoxin in outpatients, which is a common issue in outpatient settings (11). Sampling time 
errors before the drug has reached a stable state are important causes of subtherapeutic levels or inappropriate measurements in most developed countries $(15,21,22)$. Unlike the studies of Oncu et al., it was not possible to question whether sample taking was performed after reaching the steady state concentration, due to the very wide time interval scanning and inadequacies in our hospital information management system $(21,22)$. This fact is one of the limitations of our study. However, it should be noted that our study includes not only patients who use oral medication but also those who are given intravenous digoxin. Our clinical observations indicate that, the issues related to steady state were not paid attention to inpatients adequately, which was similar to the Oncu et al.'s findings. We think that awareness trainings in inpatients departments will have positive effects, similar to this study (22). Adherence problems in older patients may also make it difficult to achieve therapeutic levels $(7,23)$. In our study, the subtherapeutic level rate in the young-old group was higher than that in the other groups.

The lowest rate of patients in the therapeutic range among all elderly patients was in the veryold group. These patients had the highest toxic level rate. Ozturk and Cuhadar stated that high serum digoxin results were observed more frequently in young-old, middle-old, and very-old patients than in other age groups, and the rate was especially high in the middle-old patients (12). Limon et al. demonstrated that the mean age of patients who experienced digoxin poisoning was significantly higher than that in patients who did not experience digoxin poisoning, and the average age of patients in the study was 76 years (17). Although there are few studies claiming that there is no relationship between age and inappropriate serum digoxin measurement (21), increased toxic values with increasing age are closely related to age-related changes in pharmacokinetics and pharmacodynamics of digoxin. Muscle mass and body mass decrease in the elderly, and as a consequence, the volume of distribution for digoxin decreases with age, which results in a relatively higher serum digoxin concentration. In addition, with the decline in renal function with advancing age, the half-life of digoxin may extend to 2-3 times longer than normal $(1,7,22)$.

Similar to our study, other studies show that the number of digoxin orders among units in hospitals is the highest in the emergency departments (12, 16). In the elderly, emergency services are important because of more frequent drug intoxication and adverse effects of drugs in differential diagnosis and treatment follow-up $(9,10)$. In addition to toxicity, digoxin orders in the emergency department can be made to evaluate digoxin efficacy. Indeed, although most of our emergency department results were within the therapeutic range, unlike the results of Mundet et al., patients with levels in the subtherapeutic range comprised approximately one-third of emergency department patients, which was similar to the results of Mundet et al. (24). Interestingly, the proportion of patients with toxic levels was lower in the emergency department than in the coronary intensive care and internal medicine units, which were the other units that ordered levels most frequently. Similarly, in a study by Garcia-Iranzo et al., emergency department patients with subtherapeutic levels were more common than emergency department patients with toxic levels (14). In the coronary intensive care unit, where intravenous treatment is used extensively, it is expected that the proportion of toxic patients will be the highest (19).

In our study, approximately three-fourths of the pre-diagnoses of all orders consisted of cardiac or digoxin-related complaints. The rate of orders for appropriate indications did not differ substantially from the rates in the literature $(15,17,19)$. Past studies show that serum digoxin measurements were performed with a wide range (32\% to $88.4 \%$ ) of inappropriate criteria $(11,18,22,25)$. It has been demonstrated that inappropriate requests can be significantly reduced through in-service therapeu- 
tic drug monitoring training (22).

As a result, we evaluated serum digoxin orders in elderly patients for more than ten years. The changes in the pharmacokinetics and pharmacodynamics of digoxin in geriatric patients require physicians to be alert of subtherapeutic and toxic drug levels. Although serum digoxin concentrations may not always be a strong indicator of toxicity, they are the best available indicator and should be evaluated together with patients' clinic findings. It is appropriate to emphasize the im-

\section{REFERENCES}

1. Cheng JW, Rybak I. Use of digoxin for heart failure and atrial fibrillation in elderly patients. Am J Geriatr Pharmacother 2010;8(5):419-27. (PMID:21335295).

2. Ilerigelen B. Heart failure in elderly. Turkish Journal of Geriatrics 2010;13(Supplement 2):21-32. (in Turkish).

3. Meibohm B, Beierle I, Derendorf H. How important are gender differences in pharmacokinetics? Clin Pharmacokinet 2002;41(5):329-42. (PMID:12036391).

4. Puche Canas E, Aomar Millan I, Carrillo Santos JC. Inappropriate blood levels of digoxin in a study of 2,849 patients from a teaching hospital: the influence of age and sex. An Med Interna 2004;21(12):581-4. (PMID:15628952).

5. Ahmed A. Digoxin and reduction in mortality and hospitalization in geriatric heart failure: importance of low doses and low serum concentrations. J Gerontol A Biol Sci Med Sci 2007;62(3):323-9. (PMID:17389731).

6. Kang JS, Lee $\mathrm{MH}$. Overview of therapeutic drug monitoring. Korean J Intern Med 2009;24(1):1-10. (PMID:19270474).

7. Currie GM, Wheat JM, Kiat H. Pharmacokinetic considerations for digoxin in older people. Open Cardiovasc Med J 2011;5:130-5. (PMID:21769303).

8. Miura T, Kojima R, Sugiura Y, Mizutani M, Takatsu F, Suzuki Y. Effect of aging on the incidence of digoxin toxicity. Ann Pharmacother 2000;34(4):427-32. (PMID:10772425).

9. Hafner JW Jr, Belknap SM, Squillante MD, Bucheit KA. Adverse drug events in emergency department patients. Ann Emerg Med 2002;39(3):258-67. (PMID:11867978). portance of and raise awareness and plan periodic updated trainings for therapeutic digoxin levels in hospitals in order to promote individualized doses and optimized treatments in the elderly. There remains a great need for similar further clinical trials involving larger elderly populations.

\section{CONFLICTS OF INTEREST}

The authors declare no conflict of interest.

10. Budnitz DS, Shehab N, Kegler SR, Richards CL. Medication use leading to emergency department visits for adverse drug events in older adults. Ann Intern Med 2007;147(11):755-65. (PMID:18056659).

11. Bernard DW, Bowman RL, Grimm FA, Wolf BA, Simson MB, Shaw LM. Nighttime dosing assures postdistribution sampling for therapeutic drug monitoring of digoxin. Clin Chem 1996;42(1):45-9. (PMID:8565231).

12. Ozturk Z, Cuhadar S. Evaluation and clinical significance of factors affecting the serum digoxin levels in patients taking digoxin. Bezmialem Science 2018;6:31-6. (in Turkish).

13. Sillars A, Ho FK, Pell GP, et al. Sex differences in the association of risk factors for heart failure incidence and mortality. Heart 2020;106(3):203-12. (PMID:31366573).

14. Garcia-Iranzo EM, Rodriguez-Lucena FJ, MatosesChirivella C, Garcia-Monsalve A, Murcia-Lopez AC, Navarro-Ruiz A. Pharmacokinetic monitoring of chronic treatment with digoxin from primary health care. Farm Hosp 2017;41(4):527-32. (PMID:28683704).

15. Mordasini MR, Krahenbuhl S, Schlienger RG. Appropriateness of digoxin level monitoring. Swiss Med Wkly 2002;132(35-36):506-12. (PMID:12506332).

16. Dalaklioglu S. Evaluating appropriateness of digoxin, carbamazepine, valproic acid, and phenytoin usage by therapeutic drug monitoring. Clin Lab 2013;59(34):325-31. (PMID:23724621).

17. Limon G, Ersoy G, Oray NC, Bayram B, Limon O. Retrospective evaluation of patients with elevated digoxin levels at an emergency department. Turk J Emerg Med 2016;16(1):17-21. (PMID:27239633). 
18. Orrico KB, Wu M, Wilson AR. Assessment of the appropriateness of serum digoxin concentration measurement in a medical group setting. J Manag Care Pharm 2011;17(9):695-700. (PMID:22050394).

19. Grzesk G, Stolarek W, Kasprzak M, et al. Therapeutic drug monitoring of digoxin-20 years of experience. Pharmacol Rep 2018;70(1):184-9. (PMID:29414149).

20. Gerede DM, Koboev R, Caglar N, Kilickap M, Erol C. Evaluation and clinical significance of factors affecting the serum digoxin levels in patients taking digoxin. MN Cardiology 2015;22(1):30-6. (in Turkish).

21. Oncu S, Gelal A, Aslan O, Ucku RS. Appropriateness of digoxin measurement in hospitalized patients. Biochem Med (Zagreb) 2018;28(1):010901. (PMID:29187799)

22. Oncu S, Baskurt A, Senturk B, Aslan O, Kume T, Ucku R, Gelal A. Educational intervention to improve appropriate digoxin therapeutic drug monitoring: a quasi-experimental study. Eur J Hosp Pharm 2019;0:15 .
23. Akan P, Erdincler D, Tezcan $V$, Beger $T$. Drug use in the elderly. Turkish Journal of Geriatrics 1999;2:33-8. (in Turkish).

24. Mundet X, Vidal X, Arnau JM. The characteristics of digoxin utilization in a population under emergency care for heart failure or auricular fibrillation. Med Clin (Barc) 1993;100(3):94-7. (PMID:8426500).

25. Al Za'abi M, Al Mugbali J, Al-Waili K. Sampling time and indications appropriateness for therapeutically monitored drugs at a teaching university hospital in Oman. Saudi Pharm J 2015;23(4):458-62. (PMID:27134551). 